SCIREA Journal of Clinical Medicine

ISSN: 2706-8870

http://www.scirea.org/journal/CM

July 28, 2021

SCIREA

Volume 6, Issue 4, August 2021

\title{
Mirror self-misrecognition in dementia: caring about the person's identity
}

\section{Stephan Eyer ${ }^{1,2,3}$}

${ }^{1}$ Department of Geriatric Medicine, Centre Hospitalier du Valais Romand, Sierre

${ }^{2}$ Memory Clinic, Centre Hospitalier du Valais Romand, Sierre

${ }^{3}$ Clinical Ethics Committee, Hôpital du Valais, Sion

Correspondence address - Stephan Eyer, M.D., Hôpital du Valais - Centre Hospitalier du Valais Romand, Department of Geriatric Medicine \& Memory Clinic, 14 Rue St-Charles 3960 Sierre, SWITZERLAND, Phone: +41 (0)27 60375 00, stephan.eyer@hopitalvs.ch

Keywords: Self-misrecognition, Capgras delusion, mirror behaviors.

\begin{abstract}
Objectives and Design - This case report describes the disturbing symptom of mirror selfmisperception, a delusion known as Capgras syndrome.

Setting and Participant - A video recording captures the confusion of a demented patient who does not recognize himself in his reflected image, and instead perceives that he is looking at an identical "twin brother".
\end{abstract}


Results - It shows the delusion of misperception both in interpretation of the reflected image (mirror agnosia), and in self-recognition (autoprosopagnosia).

Conclusions and Implications - Self-misperception raises questions of how to maintain a sense of personal identity during care-giving. A person-centered and touch-based approach not only provides reassurance, but also can help preserve individual integrity and identity.

\section{Case Report}

\section{Objectives and Design}

Capgras delusion is a particular form of perception disorder. It is associated with dementia due to impairment of memory and facial recognition, as well as the presence of paranoid delusions of person substitution. Affected patients do not recognize their relatives, and believe they see duplicates (Doppelgängers) or identical-looking imposters that have replaced their loved ones. They can react with aggressiveness, fear, or indifference [1,2].

The self-misrecognition and the delusional misidentification of a "twin brother" in a mirror are rare presentations ("auto-Capgras"). Using a video recording, we demonstrate the disturbing symptom of mirror self-misrecognition in dementia. We then discuss the ethical dimensions surrounding this situation, in that self-misrecognition raises the question of how to maintain personal identity during care-giving.

\section{Setting and Participant}

We capture the confusion of a demented patient who does not recognize himself in his mirror image, and believes he is interacting with a person of identical appearance. Written consent for recording and publishing was obtained from his partner, who is his legal and healthcare representative. The pictures and the video capture are published as supplemental online material (Figures 1-4).

\section{Results}

An 80-year-old man is transferred to our geriatric rehabilitation unit after an acute respiratory infection. He has a history of moderate-to-severe, previously uninvestigated dementia. $\mathrm{He}$ walks in the hospital's hallways and perceives the reflected image of his face and body on the window. He believes that he is facing a person who looks just like him (Figure 1). He tries to touch him through the glass (Figure 2, mirror sign of Ramachandran and Binkofski) and to 
determine his exact location, thinking he might be outside on the parking lot (Figure 3). He regularly repeats these behaviors when he sees his reflection in other mirrors as well.

The video recording shows two mirror behavior disorders: 1) the phenomenon of reflection misperception, and 2) self-misperception. The patient does not discriminate the difference between his face and body from their reflections (mirror agnosia). Furthermore, he does not recognize himself in the reflecting image (autoprosopagnosia), and instead perceives a delusional "twin brother".

\section{Conclusions and Implications}

The video recording illustrates the tremendous suffering of a demented patient who does not recognize himself in his reflected image. There are some similarities between the described mirror signs and the narrative of the myth of Narcissus in Ovid's Metamorphoses: the selfnonrecognition emphasizes the loss of one's identity and the need for recognition. Unlike the ancient figure, the affected person's impaired cognitive abilities, not his lack of selfawareness, preclude any hope of future self-understanding.

The patient attempts to touch and talk to the delusional image through the glass. The observation of these mirror behaviors suggest a possible therapeutic effect of massage and touch in care-giving. Despite the severity of the cognitive impairment, implicit emotional memory is well preserved and a person-centered sensory approach provides not only reassurance, but also preserves individual integrity and identity.

\section{Figures (Pictures and Video capture)}

1. Eyer S. Picture1_Capgras [Internet]. figshare; 2019 [cited 2019Jul25].. Available from: https://figshare.com/articles/Picture1_Capgras/9063260/1

The patient believes that he is interacting with a person of identical appearance ("autoCapgras"). 


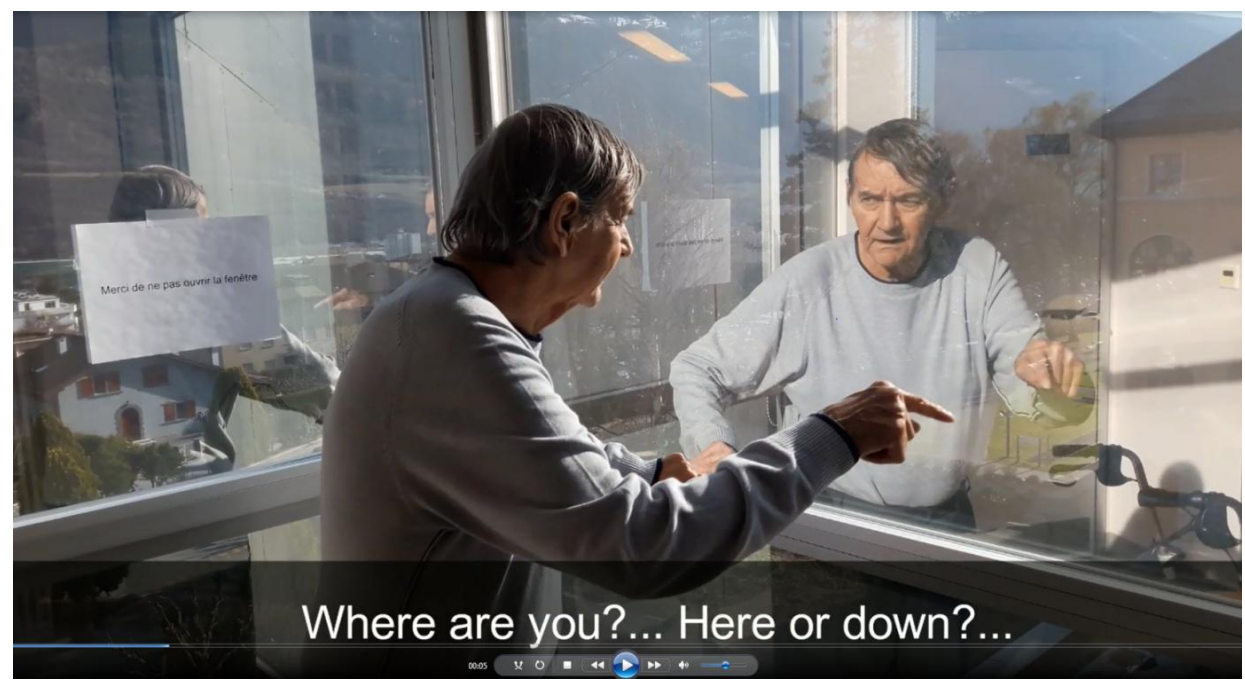

2. Eyer S. Picture2_Capgras [Internet]. figshare; 2019 [cited 2019Jul25].. Available from: https://figshare.com/articles/Picture2_Capgras/9063977/1

The patient tries to touch the delusional person through the glass (mirror sign of Ramachandran and Binkofski).

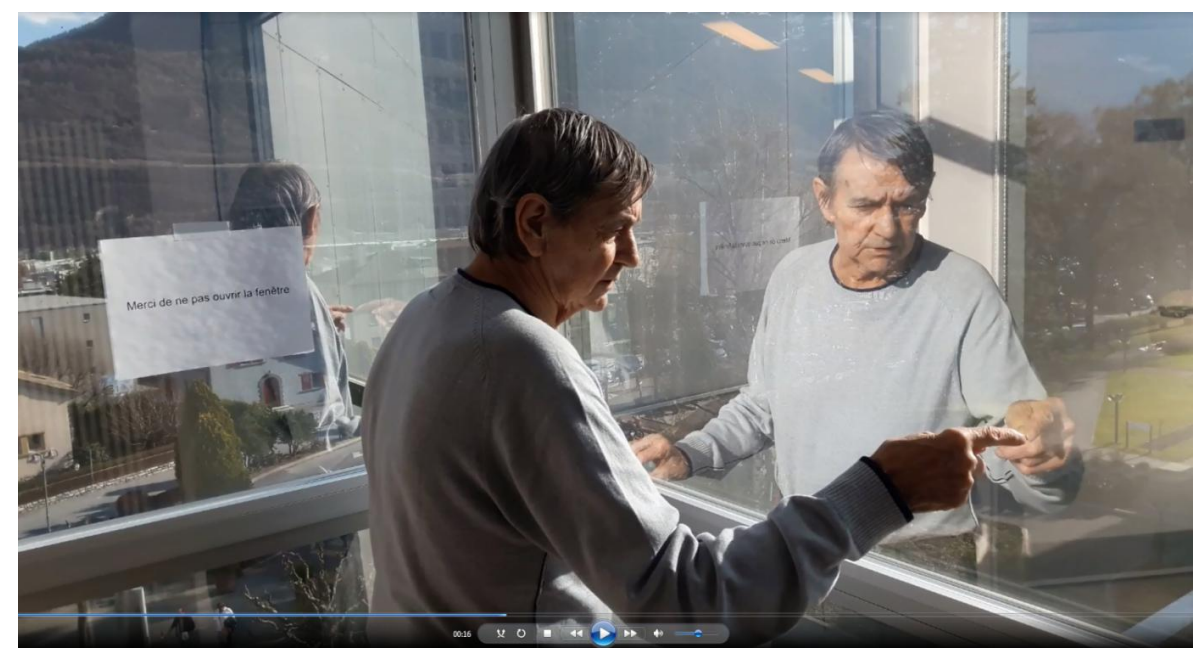

3. Eyer S. Picture3_Capgras [Internet]. figshare; 2019 [cited 2019Jul25].. Available from: https://figshare.com/articles/Picture3_Capgras/9064373/1

The patient talks to his "twin brother" and tries to find out his exact location. 


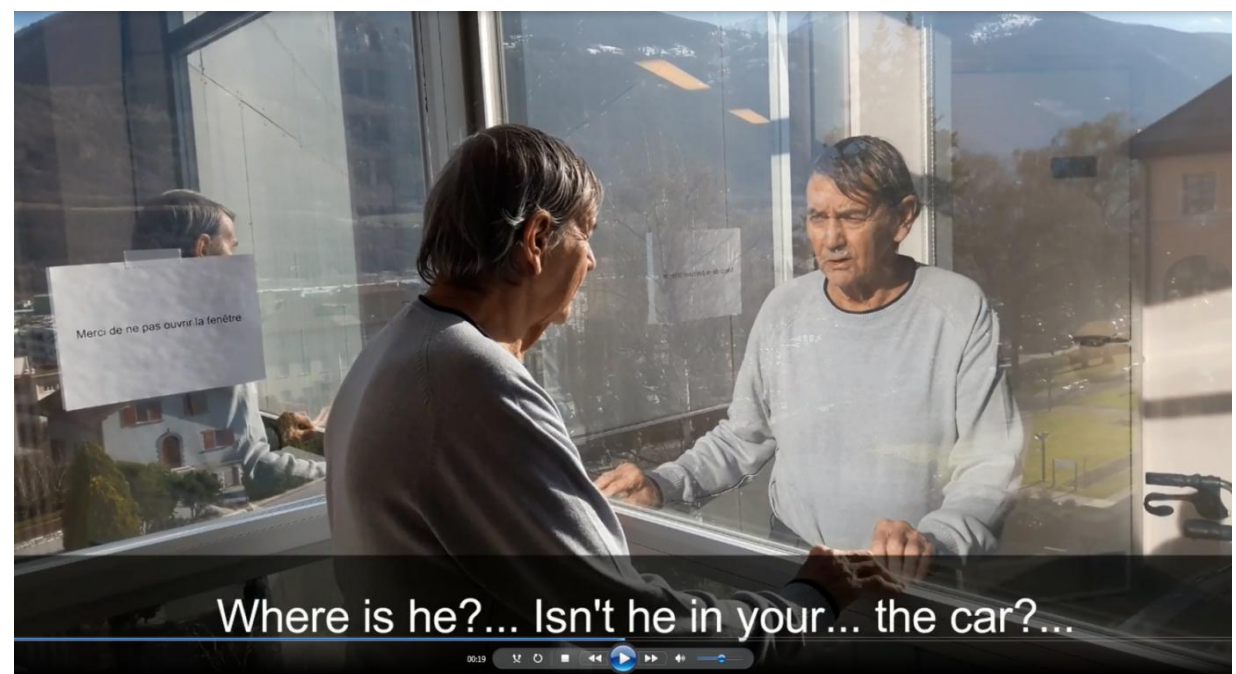

4. Eyer S. Video_Capgras.mp4 [Internet]. figshare; 2019 [cited 2019Jul25].. Available from: https://figshare.com/articles/Video_Capgras_mp4/9061778/1

The patient does not recognize himself in his mirror image (autoprosopagnosia), and instead perceives that he is looking at an identical "twin brother" ("auto-Capgras").

Availability of data - The data that support the findings of this study are openly available in Eyer, Stephan (2019): Video_Capgras.mp4. figshare. Media. To access the item, go to https://doi.org/10.6084/m9.figshare.9061778.v1

Word, reference, and graphics count - 483 words, 3 pictures, 1 video recording (supplemental online material), 2 references

Ethics approval statement - Signed consent form for recording and publishing was obtained from the legal and healthcare representative (release waiver in French).

Contributorship Statement - The authorship based on the criteria of the ICMJE is: 1) Stephan Eyer. Stephan Eyer as corresponding author had final responsability for the decision to submit for publication.

\section{References}

[1] Ghika J, Diéguez S, Assal F et al. Mirror behaviors in dementia: the many mirror signs. Rev Med Suisse. 2013 Nov 13;9(406):2095-9.

[2] Woehrle L, Ghika JA, Coutaz M et al. Capgras delusion and Alzheimer disease. Rev Med Suisse. 2018 Jun 20;14(612):1310-1313. 